\title{
Matrix Product State and mean field solutions for one-dimensional systems can be found efficiently
}

\author{
Norbert Schuch ${ }^{1,2}$ and J. Ignacio Cirac ${ }^{1}$ \\ ${ }^{1}$ Max-Planck-Institut für Quantenoptik, Hans-Kopfermann-Str. 1, D-85748 Garching, Germany. \\ ${ }^{2}$ Institute for Quantum Information, California Institute of Technology, MC 305-16, Pasadena CA 91125, U.S.A.
}

\begin{abstract}
We consider the problem of approximating ground states of one-dimensional quantum systems within the two most common variational ansatzes, namely the mean field ansatz and Matrix Product States. We show that both for mean field and for Matrix Product States of fixed bond dimension, the optimal solutions can be found in a way which is provably efficient (i.e., scales polynomially). This implies that the corresponding variational methods can be in principle recast in a way which scales provably polynomially. Moreover, our findings imply that ground states of one-dimensional commuting Hamiltonians can be found efficiently.
\end{abstract}

\section{INTRODUCTION}

Characterizing the ground states of quantum spin systems is a highly challenging task. Different from the situation for classical systems, where for the very least, the ground state can be described efficiently, this does generally not hold for quantum systems. Thus, their ground states are considerably harder to compute: as Kitaev has shown, solving this problem is likely to be hard even for quantum computers [1, 2]. Even more surprising, finding the ground states of quantum systems remains equally hard when we restrict our interest to one-dimensional systems [3]. This is in sharp contrast to the case of classical spin systems, which can be efficiently solved in one dimension, whereas two-dimensional systems are known to be NP-hard.

Despite these hardness results for ground states of quantum systems, physical properties of interest can frequently be determined efficiently using numerical methods. Even a mean field ansatz, which completely neglects correlations between the particles, may already reproduce many physical quantities relatively well [4]. In most cases however, correlations are important and other methods must be applied. While in two dimensions, imposing frustration or fermionic statistics yields Hamiltonians which cannot be assessed well by numerical methods, one-dimensional systems - despite the hardness results for 1D Hamiltonians - generally turn out to be extremely well simulatable using a method known as the the Density Matrix Renomalization Group (DMRG) algorithm [5, 6]. DMRG can be understood as a variational method over the class of Matrix Product States (MPS) 7, [8]: These states can be considered as a generalization of the mean field product states, but with a limited amount of correlations. It has been proven that this allows for the efficient approximation of ground states of gapped local Hamilonians [9].

The motivation for this work originates in the following observation: On the one side, DMRG is a highly successful algorithm which finds the correct minimum in basically all practical cases. On the other hand, it has been shown that the problem of finding MPS ground states can be NP-hard [1], as well as certain minimization problems encountered in the DMRG algorithm 10]. The contrast between these hardness results and the apparent success of mean field and MPS algorithms raises the question whether it is possible to prove the efficiency of variational methods over the class of mean-field and Matrix Product States in full generality for gapped quantum systems, or whether even studying physical 1D problems is hard, as it is the case in two dimensions.

In this paper, we settle this question by studying whether (and to which extent) for a given Hamiltonian it is possible to find the optimal mean field state or MPS for a fixed bond dimension. Surprisingly, we find that the same technique which is used to show that classical spin chains can be solved efficiently also allows for solving the problem in the case of mean field theories and MPS.

In particular, we show that it is always possible to find the optimal mean field solution of a qu- $d$-it chain of length $N$ up to accuracy $1 / \epsilon$ in energy in a time which scales as $(N / \epsilon)^{4 d}$. Concerning Matrix Product States, we find that approximating the optimal MPS of bond dimension $D$ up to precision $1 / \epsilon$ requires a computation time which scales as as $\left(N^{2 d} / \epsilon\right)^{6 D^{2}}$, where $D$ is the bond dimension of the MPS. For a fixed bond dimension, the scaling is thus polynomial both in the length of the chain and the accuracy.

Note that the result for MPS (in particular, the exponential scaling in $D$ ) is essentially optimal, since is has been shown that the difficulty of the problem has to scale exponentially with $D$ (more precisely, it is NP-hard, where $D$ is polynomial in the problem size 11]). Note also that while the exponential scaling seems daunting, in practice one is typically interested in intensive quantities for which a moderate $D$ will suffice [12]; moreover, the polynomial scaling of the algorithm in $N$ typically allows for the efficient evaluation of such quantities even in the thermodynamic limit [21].

Our findings show that variational calculations over both the mean field ansatz as well as Matrix Product States can be carried out in a way which is promised to yield the optimal solution, thus resolving the question as to whether variational algorithms over MPS such as DMRG - can be rephrased in a way which provably succeeds. Moreover, while the algorithm might be 
unpractical for $D$ 's of several 100 as used in practical DMRG implementations, the algorithm could prove useful in practice to find the optimal MPS ansatz for a low $D$, which can then be used to bootstrap DMRG [13], thus helping to avoid local minima. Finally, our findings also imply that ground states of one-dimensional commuting Hamitonians can be found efficiently, as they can be expressed as MPS.

\section{CLASSICAL SPIN CHAINS}

We start by explaining how to solve a classical spin chain efficiently. While this is known, it will help us to clarify the essential ideas of the proof technique, as used later for mean field and MPS. Given an open boundary condition (OBC) Hamiltonian $h_{k, k+1}\left(i_{k}, i_{k+1}\right), i_{k} \in$ $\{1, \ldots, d\}, k=1, \ldots, N$, we want to find the $i_{1}, \ldots, i_{N}$ which minimize the energy,

$$
E=\min _{i_{1}, \ldots, i_{N}}\left[h_{12}\left(i_{1}, i_{2}\right)+\cdots+h_{N-1, N}\left(i_{N-1}, i_{N}\right)\right] .
$$

To this end, define recursively

$$
\begin{aligned}
& E_{1}\left(i_{1}\right)=0 \\
& E_{k}\left(i_{k}\right)=\min _{i_{k-1}}\left[E_{k-1}\left(i_{k-1}\right)+h_{k-1, k}\left(i_{k-1}, i_{k}\right)\right], \quad k \geq 2 .
\end{aligned}
$$

Then, the ground state energy is given by $E=$ $\min _{i_{N}} E_{N}\left(i_{N}\right)$, and the minimization can be carried out efficiently by computing the $E_{k}\left(i_{k}\right)$ of Eq. (2) sequentially: The reason is that in every step, $E_{k}$ (the minimal energy of the half chain left of $k$ ) only depends on the value $i_{k}$ of the spin at $k$ - this is the only variable which we still need to access to minimize the energy of Hamiltonian terms to come. The computational cost is as follows: for each of the $d$ values of $i_{k}$, one has to compute $E_{k}\left(i_{k}\right)$. Each computation involves the minimization over $d$ settings of $i_{k-1}$, and the total computational cost is thus $N d^{2}$. Note that the algorithm not only yields the optimal energy, but also the corresponding ground state $i_{1}, \ldots, i_{N}$.

The intuition behind the algorithm is to proceed from left to right through the chain and at every site minimize the energy of the half chain left of $k$ as a function of the boundary setting. Here, the "boundary setting" contains all those spins whose value will still influence the optimal energy of Hamiltonian terms to the right of $k$; in the case of the classical system, this is just the spin at the boundary. Then, the optimization can be carried out sequentially by adding one new interaction at a step and minimizing the total energy of the left half chain (i.e., the previous total energy plus the new coupling) as a function of the new boundary. Sloppily speaking, the idea is that while proceeding through the chain, we have to make choices about the state, and we want to keep the dependence of the minimal energy of the half-chain on all past choices which can still influence the future.
This construction contains all important ideas for the mean field as well as for the MPS setting. For mean field, the boundary condition is again only the spin on the boundary, which however is now a continuous degree of freedom. Thus, we have to show that we can discretize its values without loosing too much accuracy. For the case of MPS, the situation seems more involved, since all choices in the past can influence the future. However, as we will show later, MPS have exactly the property that the influence of past choices on the future is fully characterized by what is passed through the bonds, and is thus bounded.

\section{MEAN FIELD}

Having understood the method, let us now consider the problem of minimizing the energy of a 1D quantum system $H=\sum_{k=1, \ldots, N-1} H_{k, k+1},\left\|H_{k, k+1}\right\|_{\infty} \leq 1$ with respect to a mean field ansatz $|\psi\rangle=\bigotimes_{k=1}^{N}\left|\psi_{k}\right\rangle,\left|\psi_{k}\right\rangle \in$ $\mathbb{C}^{d}$ (in the following, all states are normalized), where we try to minimize

$$
E=\min _{\left|\psi_{1}\right\rangle, \ldots,\left|\psi_{N}\right\rangle} \sum_{k=1}^{N-1}\left\langle\psi_{k}, \psi_{k+1}\left|H_{k, k+1}\right| \psi_{k}, \psi_{k+1}\right\rangle .
$$

This minimization again allows for a recursive formulation as in (21). However, since the parameters $\left|\psi_{k}\right\rangle$ are continuous, and the cost functions $E_{k}\left(\left|\psi_{k}\right\rangle\right)$ are nonlinear and thus cannot be solved for exactly, we restrict the minimization to an $\epsilon$-net, i.e., a discrete set of $\left|\psi_{k}^{\alpha}\right\rangle$, $\alpha=1, \ldots, \mathcal{A}$ such that

$$
\forall\left|\psi_{k}\right\rangle \in \mathbb{C}^{d} \exists \alpha:\left\|\psi_{k}-\psi_{k}^{\alpha}\right\|_{1} \leq \epsilon
$$

(we use the convention $\phi \equiv|\phi\rangle\langle\phi|$ ). As shown in 14] (Lemma II.4), such a net of size $A \leq(5 / \epsilon)^{2 d}$ exists. This reduces the algorithm to the algorithm for classical $1 D$ chains described above, which will yield the optimal solution in the set of all product states $\otimes\left|\psi_{k}^{\alpha}\right\rangle$ on the net.

The proper way to think of the net is as a constraint made on the mean field ansatz as a whole, rather than of a way to approximate each recursion step separately (since this would lead to accumulating errors). Thus, in order to bound the error made by introducing the net, we just have to bound the difference between the minimum in the set of product states and the minimum on the net in Eq. (3). To this end, start from the optimal product state $\otimes\left|\psi_{k}\right\rangle$ and replace each state by an $\epsilon$-close state $\left|\psi_{k}^{\alpha}\right\rangle$ on the net. For each term in the Hamiltonian, this yields an error of at most

$$
\begin{aligned}
& \left|\operatorname{tr}\left[H_{k, k+1}\left(\psi_{k} \otimes \psi_{k+1}-\psi_{k}^{\alpha(k)} \otimes \psi_{k+1}^{\alpha(k+1)}\right)\right]\right| \\
& \quad \leq\left\|H_{k, k+1}\right\|_{\infty}\left\|\psi_{k} \otimes \psi_{k+1}-\psi_{k}^{\alpha(k)} \otimes \psi_{k+1}^{\alpha(k+1)}\right\|_{1} \\
& \quad \leq 2 \epsilon .
\end{aligned}
$$

Thus, the total error from restricting the minimization of the energy (3) to states in the net is $2 N \epsilon$. For a targeted 
accuracy $\delta$ in energy, we thus have to choose $\epsilon=\delta / 2 N$, so that the size of each net will be $\mathcal{A}=(10 N / \delta)^{2 d}$. The minimization can be thus rewritten in the iterative form (2) and carried out in time $d^{4} \mathcal{A}^{2} N=N d^{4}(10 N / \delta)^{4 d}$.

\section{MATRIX PRODUCT STATES}

Let us now turn towards matrix product states. Starting again from a 1D quantum Hamiltonian $H=$ $\sum_{k=1}^{N-1} H_{k, k+1},\left\|H_{k, k+1}\right\|_{\infty} \leq 1$, we wish to minimize the energy over all Matrix Product States [15]

$$
\left|\chi\left(\left\{A^{k}\right\}\right)\right\rangle=\operatorname{tr}\left[A_{i_{1}}^{1} \cdots A_{i_{N}}^{N}\right]\left|i_{1}, \ldots, i_{N}\right\rangle
$$

of a given bond dimension $D, A_{i}^{k} \in M_{D \times D}$ (except for $A_{i}^{1} \in M_{1 \times D}, A_{i}^{N} \in M_{D \times 1}$ ). It is known (cf. [15]) that every MPS can be brought to a standard form for which $\sum_{i} A_{i}^{k}\left(A_{i}^{k}\right)^{\dagger}=\mathbb{1}$. With this gauge, $\left|\chi\left(\left\{A^{k}\right\}\right)\right\rangle$ is normalized, and the energy of a term $H_{k, k+1}$ can be computed as follows: Define

$$
\begin{aligned}
\rho_{1} & =1, \\
\rho_{k+1}=\mathcal{R}\left(A_{k}, \rho_{k}\right) & :=\sum_{i}\left(A_{i}^{k}\right)^{\dagger} \rho_{k} A_{i}^{k} .
\end{aligned}
$$

Then, the energy of $H_{k, k+1}$ is given by

$$
\begin{aligned}
& \mathcal{E}_{k}\left(A^{k}, A^{k+1}, \rho_{k}\right)= \\
& \sum_{a, b, c, d}\left\langle a, b\left|H_{k, k+1}\right| c, d\right\rangle \operatorname{tr}\left[\left(A_{a}^{k+1}\right)^{\dagger}\left(A_{b}^{k}\right)^{\dagger} \rho_{k} A_{c}^{k} A_{d}^{k+1}\right] .
\end{aligned}
$$

The task is to minimize the total energy over the set of MPS,

$$
E=\min _{A^{1}, \ldots, A^{N}} \sum_{k=1}^{N-1} \mathcal{E}_{k}\left(A^{k}, A^{k+1}, \rho_{k}\right) .
$$

Note that by virtue of the recursion relation (4), $\mathcal{E}_{k}$ actually depends on all $A^{l}$ with $l \leq k+1$, thus seemingly ruling out the same approach as for the mean field ansatz.

To resolve this, we rewrite the minimization over all $A_{k}$ in (6) as a series of constrained minimizations,

$\min _{A_{1}, \ldots, A_{N}}=\min _{A_{N-1}, A_{N}, \rho_{N-1}} \cdots \min _{\left(A_{k-1}, \rho_{k-1}\right) \rightarrow \rho_{k}} \cdots \min _{\left(A_{1}, \rho_{1} \equiv 1\right) \rightarrow \rho_{2}}$

where $\left(A_{k-1}, \rho_{k-1}\right) \rightarrow \rho_{k}$ denotes the tuples $\left(A_{k-1}, \rho_{k-1}\right)$ which are compatible with $\rho_{k}$, i.e., $\mathcal{R}\left(A_{k-1}, \rho_{k-1}\right)=\rho_{k}$. Based on this rephrased form of the minimization ([6), we define

$$
\begin{aligned}
& E_{k}\left(A^{k+1}, \rho_{k+1}\right)= \\
& \min _{\left(A_{k}, \rho_{k}\right) \rightarrow \rho_{k+1}} \mathcal{E}_{k}\left(A^{k}, A^{k+1}, \rho^{k}\right)+E_{k-1}\left(A^{k}, \rho_{k}\right)
\end{aligned}
$$

(if the minimum is over an empty set, let $E_{k}=+\infty$ ), with $E_{0} \equiv 0$. Then, we can sequentially solve for the $E_{k}$, always only keeping track of them as a function of $A^{k}$ and $\rho_{k}$ at the boundary, and thus solve the minimization problem (6).

Clearly, it will again be necessary to use nets to be able to implement the optimization efficiently. We will put a net on both the $A$ 's and the $\rho$ 's, and denote elements of the nets by $\tilde{A}$ and $\tilde{\rho}$. Let us define an operation $\mathcal{N}$ : $\rho \mapsto \tilde{\rho}$ which maps every $\rho$ to the closest point in the net. We define a netified recursion relation for the $\tilde{\rho}$ 's, $\tilde{\mathcal{R}}=\mathcal{N} \circ \mathcal{R}$, and define all minimizations (in particular the constrained minimizations $\tilde{E}_{k}$ ) with respect to variables $\tilde{A}^{k}$ and $\tilde{\rho}_{k}$ in the net, and constraints according to the netified recursion relation $\tilde{\mathcal{R}}$ [22].

This coarse-grained version of the iterated protocol, which can be carried out efficiently, will give the optimal solution in an ansatz class which is defined by the coarse-grained variables and the coarse-grained recursion relation - note that this is not equal to the optimal solution with respect to the coarse-grained MPS due to the additional coarse-graining $\mathcal{N}$ in the the $\rho$ 's.

To bound the error made by introducing the nets, we compute how much the energy of an arbitrary MPS changes due to the coarse-graining of the $A$ 's and the $\rho$ 's. To this end, we first bound the difference in energy caused by coarse-graining the $\rho$ 's as compared to the energy of the MPS described by the same $\tilde{A}^{k}$ 's, and second, we bound the error in energy made by coarse-graining the $A^{k}$ 's. By choosing the nets such that both of these errors become small, we make sure that the $\tilde{A}^{k}$ 's found by optimizing the above recursion relations yield an MPS which is close in energy to the optimal MPS.

Let us first bound the error made by inserting $\mathcal{N}$. We will put an $\epsilon_{\rho}$-net on the $\rho$ 's, i.e. for each $\rho$ there is a $\tilde{\rho}$ in the net with $\|\rho-\tilde{\rho}\|_{1} \leq \epsilon_{\rho}$. From (4),

$$
\begin{aligned}
\| \tilde{\rho}_{k}- & \rho_{k} \|_{1}= \\
& =\left\|\mathcal{N}\left(\sum\left(\tilde{A}_{i}^{k}\right)^{\dagger} \tilde{\rho}_{k-1} \tilde{A}_{i}^{k}\right)-\sum\left(\tilde{A}_{i}^{k}\right)^{\dagger} \rho_{k-1} \tilde{A}_{i}^{k}\right\|_{1} \\
& =\left\|\sum\left(\tilde{A}_{i}^{k}\right)^{\dagger}\left(\tilde{\rho}_{k-1}-\rho_{k-1}\right) \tilde{A}_{i}^{k}+\epsilon_{\rho} \sigma\right\|_{1} \quad\left(\|\sigma\|_{1} \leq 1\right) \\
& \leq\left\|\sum \tilde{A}_{i}^{k}\left(\tilde{A}_{i}^{k}\right)^{\dagger}\left(\tilde{\rho}_{k-1}-\rho_{k-1}\right)\right\|_{1}+\epsilon_{\rho} \\
& \leq\left\|\tilde{\rho}_{k-1}-\rho_{k-1}\right\|_{1}+\epsilon_{\rho} \leq \cdots \\
& \leq(k-1) \epsilon_{\rho} .
\end{aligned}
$$

To bound the effect of the error in $\rho^{k}$ on each Hamiltonian term in Eq. (5), note that (5) can be rewritten as

$$
\mathcal{E}_{k}\left(\tilde{A}^{k}, \tilde{A}^{k+1}, \rho_{k}\right)=\operatorname{tr}\left[V\left(H_{k, k+1} \otimes \mathbb{1}\right) V^{\dagger} \rho_{k}\right]
$$

with an isometry $V_{\alpha, a b \beta}=\left(\tilde{A}_{a}^{k} \tilde{A}_{b}^{k+1}\right)_{\alpha \beta}$, $\sum_{a, b, \beta} V_{\alpha, a b \beta} \bar{V}_{\alpha^{\prime}, a b \beta}=\delta_{\alpha, \alpha^{\prime}}$. Then,

$$
\begin{aligned}
\left\|\mathcal{E}_{k}\left(\tilde{A}^{k}, \tilde{A}^{k+1}, \tilde{\rho}_{k}\right)-\mathcal{E}_{k}\left(\tilde{A}^{k}, \tilde{A}^{k+1}, \rho_{k}\right)\right\|_{1}= \\
\quad=\left\|\operatorname{tr}\left[V\left(H_{k, k+1} \otimes \mathbb{1}\right) V^{\dagger}\left(\tilde{\rho}_{k}-\rho_{k}\right)\right]\right\|_{1} \\
\quad \leq\left\|V\left(H_{k, k+1} \otimes \mathbb{1}\right) V^{\dagger}\right\|_{\infty}\left\|\tilde{\rho}_{k}-\rho_{k}\right\|_{1} \\
\quad \leq(k-1) \epsilon_{\rho} .
\end{aligned}
$$


Thus, the total error in energy due to the net on the $\rho$ 's is

$$
\delta_{\rho}=\sum_{k=1}^{N-1}(k-1) \epsilon_{\rho} \leq \frac{1}{2} N^{2} \epsilon_{\rho} .
$$

Second, we have to bound the error in energy made by replacing the $A^{k}$ by $\tilde{A}^{k}$ chosen from an $\epsilon_{A}$-net, which approximates the $A_{k}$ in operator norm up to $\epsilon_{A}$. To this end, define $\Delta^{k}=\tilde{A}^{k}-A^{k}$ and $\mathbb{E}_{k}(\rho)=\sum_{i} A_{i}^{k} \rho\left(A_{i}^{k}\right)^{\dagger}$, $\mathbb{D}_{k}(\rho)=\sum_{i} A_{i}^{k} \rho\left(\Delta^{k}\right)^{\dagger}$. Then, $\mathbb{E}_{k}(\mathbb{1})=\mathbb{1}, \mathbb{E}_{k}$ is contractive with respect to $\|\cdot\|_{\infty}$, and

$$
\left\|\mathbb{D}_{k}(\rho)\right\|_{\infty} \leq \sum_{i}\left\|A_{i}^{k}\right\|_{\infty}\|\rho\|_{\infty}\left\|\Delta_{i}^{k}\right\|_{\infty} \leq d \epsilon_{A}\|\rho\|_{\infty}
$$

The overlap of the MPS with and without a net is given by

$$
\left\langle\chi\left(\left\{A^{k}\right\}\right) \mid \chi\left\{\tilde{A}^{k}\right\}\right\rangle=\left(\left(\mathbb{E}_{1}+\mathbb{D}_{1}\right) \circ \cdots \circ\left(\mathbb{E}_{N}+\mathbb{D}_{N}\right)\right)(\mathbb{1}) .
$$

For the iterated application of $\left(\mathbb{E}_{k}+\mathbb{D}_{k}\right)$, we use that the deviation $\sigma_{k}$ from the identity grows according to

$$
\begin{aligned}
\left\|\sigma_{k-1}\right\|_{\infty} & =\left\|\left(\mathbb{E}_{k}+\mathbb{D}_{k}\right)\left(\mathbb{1}+\sigma_{k}\right)-\mathbb{1}\right\|_{\infty} \\
& \leq\left\|\mathbb{E}_{k}\left(\sigma_{k}\right)\right\|_{\infty}+\left\|\mathbb{D}_{k}(\mathbb{1})\right\|_{\infty}+\left\|\mathbb{D}_{k}\left(\sigma_{k}\right)\right\|_{\infty} \\
& \leq\left(1+d \epsilon_{A}\right)\left\|\sigma_{k}\right\|_{\infty}+d \epsilon_{A} .
\end{aligned}
$$

Under the condition that $\left\|\sigma_{N-k}\right\|_{\infty} \leq 2 k d \epsilon_{A} \leq 1$, this yields $\left|\left\langle\chi\left(\left\{A^{k}\right\}\right) \mid \chi\left\{\tilde{A}^{k}\right\}\right\rangle\right| \geq 1-2 N d \epsilon_{A}$. From this, we can derive a bound on the difference in energy,

$$
\begin{aligned}
\delta_{A} & =\left|\operatorname{tr}\left[H\left(\chi\left(\left\{A^{k}\right\}\right)-\chi\left(\left\{\tilde{A}^{k}\right\}\right)\right)\right]\right| \\
& \leq \mid H\left\|_{\infty}\right\| \chi\left(\left\{A^{k}\right\}\right)-\chi\left(\left\{\tilde{A}^{k}\right\}\right) \|_{1} \\
& \leq 2 N \sqrt{1-\left|\left\langle\chi\left(\left\{A^{k}\right\}\right) \mid \chi\left\{\tilde{A}^{k}\right\}\right\rangle\right|^{2}} \\
& \leq 4 N^{3 / 2} \sqrt{d \epsilon_{A}} .
\end{aligned}
$$

Let the targeted error in energy now be $\delta$, and choose $\delta_{A}=\delta_{\rho}=\delta / 2$. Thus, we will need nets of precision $\epsilon_{\rho}=\delta / N^{2}$ and $\epsilon_{A}=\delta^{2} / 64 N^{3} d$, respectively. Such nets exist of size $\mathcal{A}_{\rho}=\left(3 / \epsilon_{\rho}\right)^{D^{2}}$ and $\mathcal{A}_{A}=\left(3 / \epsilon_{A}\right)^{2 d D^{2}}$ ([16], see Appendix). Each evaluation of an energy in (5) takes $d^{4} D^{3}$ elementary steps, and thus the total computational cost is

$$
N d^{4} D^{3}\left[\frac{3^{2 d+1} 2^{12 d} N^{6 d+2} d^{2 d}}{\delta^{3}}\right]^{2 D^{2}}
$$

\section{COMMUTING HAMILTONIANS}

Our results also imply that the ground state of a local Hamiltonian on a one-dimensional chain with mutually commuting terms can be found efficiently. This follows straight away from the fact that the ground state of any commuting Hamiltonian can be expressed as an MPS with bond dimension $D=d^{2}$ [17].
Alternatively, one can map the problem of solving any one-dimensional commuting Hamiltonian to solving a classical 1D chain, as shown by Bravyi and Vyalyi [18], which allows to use the classical 1D algorithm described at the beginning. (In fact, they show that every commuting 2-local Hamiltonian - which is always the case in 1D - can be mapped to a classical problem on the same interaction graph.)

\section{PERIODIC BOUNDARY CONDITIONS}

Up to now, we have focused on Hamiltonians on OBC $1 \mathrm{D}$ chains. Let us now briefly discuss how to adapt our method to the case of a periodic boundary condition (PBC) 1D system.

In the case of both classical chains and the mean field ansatz, the PBC case can be tackled by additionally letting all $E_{k}$ in (2) depend on $i_{1}\left(\left|\psi_{1}^{\alpha}\right\rangle\right)$ : the reason is that $i_{1}$ is also part of the half-chain boundary, as the value of $i_{1}$ will influence the optimal energy when evaluating the Hamiltonian term $h_{N, 1}$.

To solve a PBC Hamiltonian with (OBC) MPS, the situation is a bit more subtle, as the energy of the coupling $H_{N, 1}$ does not only depend on $A^{1}$, but also on the way in which it is passed through the chain. Two possibilities to deal with that would either be to evaluate the $E_{k}$ not only as a function of $A^{k}$ and $\rho_{k}$, but also of the $\rho_{k}^{\alpha \beta}$ arising from putting an operator basis $|\alpha\rangle\langle\beta|$ at site 1 [i.e., starting the recursion from the state $\left.\rho_{2}^{\alpha \beta}=\left(A_{\alpha}^{1}\right)^{\dagger} A_{\beta}^{1}\right]$; or to keep the dependence on $A^{1}$ and $A^{k}$, and instead of keeping the dependence on $\rho_{k}$ rather make $E_{k}$ depend on the possible channels $\mathcal{R}\left(A^{k}, \mathcal{R}\left(A^{k-1}, \cdots, \mathcal{R}\left(A^{2}, \cdot\right)\right)\right)$ passing $\rho_{1}$ through the MPS. A more efficient way to deal with PBC Hamiltonians is however to fold the chain: This yields an OBC Hamiltonian on a chain of length $N / 2$ which can be solved with the original algorithm using an OBC MPS with bond dimension $D^{2}$, which includes the case of the folded MPS of dimension $D$. Similarly, the case of PBC MPS with bond dimension $D$ can be solved by embedding it in an OBC MPS with bond dimension $D^{2}$.

\section{Acknowledgements}

We thank B. Horstmann, A. Kay, D. Pérez-García, and K.G. Vollbrecht for helpful discussions and comments. This work has been supported by the EU (QUEVADIS, SCALA), the German cluster of excellence project MAP, the Gordon and Betty Moore Foundation through Caltech's Center for the Physics of Information, and the National Science Foundation under Grant No. PHY0803371.

Note added: Similar results have been independently obtained by D. Aharonov, I. Arad, and S. Irani [20]. 
Nets

We use [16], Lemma 4.10. It states that in $\mathbb{R}^{n}$ with any norm ||$|\cdot| \|$, there exists an $\epsilon$-net with respect to $|\|\cdot|\||$ for the unit sphere with cardinality at most $(1+2 / \epsilon)^{n} \leq$ $(3 / \epsilon)^{n}$.

This Theorem can be applied directly to the case of $D \times D$ density operators which span a $D^{2}$-dimensional real vector space. The size of an $\epsilon_{\rho}$-net with respect to the trace norm is thus $\mathcal{A}_{\rho} \leq\left(3 / \epsilon_{\rho}\right)^{D^{2}}$. To create such a net, one can e.g. place a lattice on $\mathbb{R}^{D^{2}}$ and use that the trace norm is bounded relative to the Euclidean distance.

For the case of the space of isometries $A: \mathbb{C}^{D} \rightarrow$ $\mathbb{C}^{d} \times \mathbb{C}^{D}$, it is necessary to embed them in a vector space, namely the space of all complex $D \times d D$ matrices. Then, the theorem states that there is a net of isometries of size at most $\mathcal{A}_{A} \leq\left(3 / \epsilon_{A}\right)^{2 d D^{2}}$. (Assuming that the net consists of unitaries imposes no restriction, since the theorem bounds the size of any maximal set of vectors with distance $\geq \epsilon$.) By utilizing the Solovay-Kitaev theorem, we can generate such a net efficiently for any $D[1,19]$.
[1] A. Y. Kitaev, A. H. Shen, and M. N. Vyalyi, Classical and quantum computation (American Mathematical Society, Providence, Rhode Island, 2002).

[2] D. Aharonov and T. Naveh (2002), quant-ph/0210077.

[3] D. Aharonov, D. Gottesman, S. Irani, and J. Kempe, Commun. Math. Phys. 287, 41 (2009).

[4] P. M. Chaikin and T. C. Lubensky, Principles of Condensed Matter Physics (Cambridge University Press, Cambridge, 1995).

[5] S. R. White, Phys. Rev. Lett. 69, 2863 (1992).

[6] U. Schollwöck, Rev. Mod. Phys. 77, 259 (2005).

[7] S. Östlund and S. Rommer, Phys. Rev. Lett. 75, 3537 (1995).

[8] F. Verstraete, D. Porras, and J. I. Cirac, Phys. Rev. Lett. 93, 227205 (2004).

[9] M. Hastings, J. Stat. Mech. p. P08024 (2007).

[10] J. Eisert, Phys. Rev. Lett. 97, 260501 (2006).

[11] N. Schuch, I. Cirac, and F. Verstraete, Phys. Rev. Lett. 100, 250501 (2008).

[12] F. Verstraete and J. I. Cirac, Phys. Rev. B 73, 094423 (2006).

[13] M. Hastings, private communication.

[14] P. Hayden, D. Leung, P. W. Shor, and A. Winter, Commun. Math. Phys. 250, 371 (2004).

[15] D. Perez-Garcia, F. Verstraete, M. M. Wolf, and J. I. Cirac, Quant. Inf. Comput. 7, 401 (2007).

[16] G. Pisier, The volume of convex bodies and Banach space geometry (Cambridge University Press, Cambridge, 1989).

[17] M. M. Wolf, F. Verstraete, M. B. Hastings, and J. I. Cirac, Phys. Rev. Lett. 100, 070502 (2008).

[18] S. Bravyi and M. Vyalyi, Quant. Inf. Comput. 5, 187
(2005).

[19] A. Harrow, Bachelor Thesis. MIT, 2001. http://phm.cba.mit.edu/Theses/01.05.aram.pdf

[20] D. Aharonov, I. Arad, and S. Irani, Phys. Rev. A 82, 012315 (2010); arXiv:0910.5055

[21] In condensed matter problems, one is often interested in the value of intensive quantities in the thermodynamic limit, $N \rightarrow \infty$. By invoking scaling properties typically found in practical situations [5, 6], one can otain the scaling of the computation time as a function of the desired precision $\delta$ for quantities like the energy density $e(\infty)=$ $\lim E(N) / N$ as follows: Generally, the error made in estimating $e(\infty)$ goes as $|e(\infty)-E(N) / N|=\operatorname{poly}(1 / N)$. Thus, in order to compute $e(\infty)$ with accuracy $\delta$, we need to take $N=\operatorname{poly}(1 / \delta)$. In order to obtain a $\delta$ approximation of a ground state by an MPS, we need a bond dimension of at most $D=\operatorname{poly}(N / \delta)$, whereas for gapped Hamiltonians, $D=\operatorname{poly}(\log (N / \delta))$ will typically suffice. Combining that with (7), we find that the computation time needed to compute an intensive quantity in the thermodynamic limit with precision $\delta$ scales at most as $(1 / \delta)^{\text {poly }(1 / \delta)}$, whereas for gapped systems, a quasipolynomial scaling $(1 / \delta)^{\text {poly }(\log (1 / \delta))}$ can be expected. In particular, the latter result shows that for gapped Hamiltonians, we can generally evaluate intensive quantities in the thermodynamic limit in a way which scales quasipolynomially in the desired accuracy.

[22] Note that we never actually have to evaluate $\mathcal{N}$ : To compute $\tilde{E}_{k}\left(\tilde{A}_{k}, \tilde{\rho}_{k}\right)$, we loop over all $\tilde{A}_{k-1}, \tilde{\rho}_{k-1}$ in the net and check whether $\left\|\mathcal{R}\left(\tilde{A}_{k-1}, \tilde{\rho}_{k-1}\right)-\tilde{\rho}_{k}\right\|_{1} \leq \epsilon$. 